\title{
A SURVEY ON SINGULAR SOLUTIONS OF FIRST-ORDER PARTIAL DIFFERENTIAL EQUATIONS
}

\author{
By Shyuichi Izumiya, Bing Li and Jianming Yu
}

\begin{abstract}
This is a summary of the papers $[9,10,11,12,13,15]$, in which we mainly studied singular solutions of first order differential equations.
\end{abstract}

\section{Introduction}

How should we define the notion of singular solutions for differential equations? In $[9,10,11,12,13,15]$ we have considered this philosophical and stimulating problem for first order differential equations. The first example of singular solutions was discovered by Brook Tayler about 290 years ago (cf. [7]). 20 years after that Alex Claude Clairaut studied a class of equations which have singular solutions [2]. This equation is called the Clairaut equation now: $y=x \cdot \frac{d y}{d x}+f\left(\frac{d y}{d x}\right)$. It has a quite beautiful geometric structure as follows : There exists a "general solution" that consists of lines ; $y=t \cdot x+f(t)$, where $t$ is a parameter and the singular solution is the envelope of such a family. In classical treatises of equations (Carathéodory [1] , Courant-Hilbert [3], Forsyth [4] [5], Ince [7], Petrovski [17]) the discussions of equations with singular solutions are informal. In these, a "general solution" of a differential equation is defined to be an one-parameter family of solutions and a "singular solution" is a solution which is not contained in the "general solution". However, this definition of singular solutions is very confused as the following example shows:

Example 0.1. Consider the equation $y=2 p \cdot x-p^{2}$, where $p=\frac{d y}{d x}$. In [7] the "general solution" is given by

$$
\left\{\begin{array}{l}
x=\frac{c}{p^{2}}+\frac{2}{3} p \\
y=2 p \cdot x-p^{2}
\end{array}\right.
$$

where $c$ is a parameter. It is clear that $y=0$ is also a solution, but it is not contained in the "general solution". Then $y=0$ must be the "singular solution". On the other hand, we have a two parameter family of solutions :

$$
\gamma_{\left(c_{1}, c_{2}\right)}(t)=(x, y, p)=\left(\frac{2}{3} c_{1} e^{-t}+c_{2} e^{2 t}, \frac{1}{3} c_{1}^{2} e^{-2 t}+2 c_{1} c_{2} e^{t}, c_{1} e^{-t}\right) .
$$

If we fix $p(0)=c_{1} \neq 0$ and put $c=c_{2} c_{1}^{2}$, then we have $x=\frac{2}{3} p+\frac{c}{p^{2}}$ and $y=2 p \cdot x-p^{2}$. But, if we fix $p(0)=c_{1}=0$, then we have $y=0$. Moreover, if we consider this family of

Received May 18, 1993. 
solutions around a point $\left(x_{0}, 0,0\right), x_{0} \neq 0$, then we have the relation $x(0)=\frac{2}{3} c_{1}+c_{2}=x_{0}$, so that, we have an one-parameter family of "solutions" around $\left(x_{0}, 0,0\right)$ :

$$
\gamma_{c_{1}}(t)=\left(\frac{2}{3} c_{1} e^{-t}+\left(x_{0}-\frac{2}{3} c_{1}\right) e^{2 t}, \frac{1}{3} c_{1}^{2} e^{-2 t}+2 c_{1}\left(x_{0}-\frac{2}{3} c_{1}\right) e^{t}, c_{1} e^{-t}\right)
$$

Of course, $y=0$ is contained in this family of solutions.

Even in modern articles $([8,14])$, they are studied under special assumptions.

In this note we give a survey on our works $[9,10,11,12,13,15]$ that attempt to give a rigorous definition of the notion of singular solutions of first order differential equations. In [6] M. and T. Fukuda tried to give a rigorous definition of singular solutions of higher order ordinary differential equations. Their definition is nearly correct, however the definition of singular solutions is strongly dependent on the definition of general solutions. The vagueness of the definition of singular solutions is caused by that of general solutions, so that we should begin to define "general solutions" in the correct way. In conclusion we do not have a correct rigorous definition for general first order differential equations (cf. Example 3.4). However, we established the notion of singular solutions for completely integrable first order differential equations (cf. Theorems 2.5, 3.2, 3.5, Corollaries 2.7, 3.3).

\section{Framework}

The most general framework to consider first order differential equation is given in the framework of contact geometry, however all arguments should be understood locally, so that we may consider the 1 -jet space $J^{1}\left(\mathbf{R}^{n}, \mathbf{R}\right)$ of functions of $n$-variables by the well-known Darboux's theorem. A system of first order differential equation is most naturally interpreted as being a closed subset of $J^{1}\left(\mathbf{R}^{n}, \mathbf{R}\right)$. Unless the contrary is specifically stated, we use the following definition. A first order differential equation (or briefly, an equation) is a submanifold germ $\left(E^{r}, z_{0}\right) \subset J^{1}\left(\mathbf{R}^{n}, \mathbf{R}\right)$ of dimension $r$ in the 1-jet space of functions of $n$-variables, where $n+1 \leq r \leq 2 n$. It is called $a$ holonomic system if $r=n+1$ and a single equation if $r=2 n$. Let $\theta$ be the canonical contact form on $J^{1}\left(\mathbf{R}^{n}, \mathbf{R}\right)$ which is given by $\theta=d y-\sum_{i=1}^{n} p_{i} d x_{i}$, where $(x, y, p)$ are canonical coordinates of $J^{1}\left(\mathbf{R}^{n}, \mathbf{R}\right)$. We define a geometric solution of $\left(E^{r}, z_{0}\right)$ to be an $n$ dimensional submanifold $i:\left(L, z_{0}\right) \subset J^{1}\left(\mathbf{R}^{n}, \mathbf{R}\right)$ such that $\theta \mid L=0$ and $L \subset\left(E^{r}, z_{0}\right)$ (i.e. a Legendrian submanifold which is contained in $\left.\left(E^{r}, z_{0}\right)\right)$. For a Legendrian submanifold $i:\left(L, z_{0}\right) \subset J^{1}\left(\mathbf{R}^{n}, \mathbf{R}\right), z_{0} \in L$ is said to be a Legendrian singular point if $\pi \circ i$ is not an immersion at $z_{0}$. A geometric solution $i:\left(L, z_{0}\right) \subset J^{1}\left(\mathbf{R}^{n}, \mathbf{R}\right)$ of $\left(E^{r}, z_{0}\right)$ is called classical if $z_{0}$ is not a Legendrian singular point (i.e., a Legendrian regular point). We remark that a geometric solution is equal to the image of $j^{1} f:\left(\mathbf{R}^{n}, 0\right) \rightarrow J^{1}\left(\mathbf{R}^{n}, \mathbf{R}\right)$ for a smooth function germ $f:\left(\mathbf{R}^{n}, 0\right) \rightarrow \mathbf{R}$ if and only if $i$ is classical.

We also consider singularities of equations. We say that $z \in\left(E^{r}, z_{0}\right)$ is a contact singular point if $\theta\left(T_{z} E^{r}\right)=0$. We denote the set of contact singular points by $\Sigma_{c}\left(E^{r}\right)$. A point $z \in\left(E^{r}, z_{0}\right)$ is called a $\pi$-singular point if $\operatorname{rank} d(\pi \mid E)_{z}<n$. We denote the set of $\pi$-singular points by $\Sigma_{\pi}\left(E^{r}\right)$ and $\pi\left(\Sigma_{\pi}\left(E^{r}\right)\right)=D_{E^{r}}$, where $\pi(x, y, p)=(x, y)$. We call the set $D_{E^{r}}$ the discriminant set of the equation $\left(E^{r}, z_{0}\right)$.

In order to define the notion of singular solutions, we need to define the notion of complete solutions. An equation $\left(E^{r}, z_{0}\right)$ is said to be completely integrable if there 
exists a foliation by geometric solutions on $\left(E^{r}, z_{0}\right)$. In this case such a foliation is called a complete solution of $\left(E^{r}, z_{0}\right)$. We say that a complete solution of $\left(E^{r}, z_{0}\right)$ is classical if each geometric solution in the complete solution is classical.

We now define the notion of singular solutions. Let $i:\left(L, z_{0}\right) \subset J^{1}\left(\mathbf{R}^{n}, \mathbf{R}\right)$ be a geometric solution of $\left(E^{r}, z_{0}\right)$. We say that $i:\left(L, z_{0}\right) \subset J^{1}\left(\mathbf{R}^{n}, \mathbf{R}\right)$ is a singular solution in the wider sense, if it cannot be embedded into any complete solution. We also say that it is a singular solution in the strict sense, if there exists an open neighbourhood $U$ of $z_{0}$ such that for each $z_{1} \in U$, the germ $i:\left(L, z_{1}\right) \subset J^{1}\left(\mathbf{R}^{n}, \mathbf{R}\right)$ cannot be embedded into any complete solutions. We remark that any geometric solution $i:\left(L, z_{0}\right) \subset J^{1}\left(\mathbf{R}^{n}, \mathbf{R}\right)$ of $\left(E^{r}, z_{0}\right)$ is a singular solution in the wider sense if the equation is not completely integrable. Since the equation of Example 0.1 is not completely integrable (cf. Theorem $2.6)$, the solution $y=0$ is a singular solution in the wider sense.

We say that an equation $\left(E^{r}, z_{0}\right)$ is involutory at $z \in\left(E^{r}, z_{0}\right)$ if there is a Legendrian submanifold $L$ tangent to $E^{r}$ at $z$. We also say that an equation $\left(E^{r}, z_{0}\right)$ is involutory if it is involutory at any point of $\left(E^{r}, z_{0}\right)$. Since single equations are automatically involutory, the notion of involutory is essential for overdetermined systems of first order partial differential equations (cf. $[6,7])$.

\section{Singular solutions in the strict sense}

In [9] completely integrable equations were studied by using the theory of Legendrian unfoldings and the following fact was discovered.

Proposition 2.1. For an equation with classical complete solution $\left(E^{r}, z_{0}\right)$, the contact singular set $\Sigma_{c}\left(E^{r}\right)$ is an n-dimensional submanifold of $\left(E^{r}, z_{0}\right)$ if it is nonempty.

Since the contact singular set $\Sigma_{c}\left(E^{r}\right)$ is a contact invariant set, we have the following corollary.

COROLlaRY 2.2. For a completely integrable first order equation $\left(E^{r}, z_{0}\right)$, the contact singular set $\Sigma_{c}\left(E^{r}\right)$ is an n-dimensional submanifold of $\left(E^{r}, z_{0}\right)$ if it is non-empty.

By definition, if $\Sigma_{c}\left(E^{r}\right)$ is an $n$-dimensional submanifold of $\left(E^{r}, z_{0}\right)$ then it is automatically a geometric solution of $\left(E^{r}, z_{0}\right)$.

The following is a classical example of an equation with singular solution.

Example 2.3. The Clairaut equation : $y=x \cdot p+f(p)$. The singular solution is $\Sigma_{c}\left(E^{2}\right)=\Sigma_{\pi}\left(E^{2}\right)$ and the discriminant set $D_{E^{2}}$ is the envelope of the complete solution $y=x \cdot c+f(c)$.

In classical treatises, it has been considered that $\Sigma_{\pi}\left(E^{r}\right)$ was a strong candidate for the singular solution like in the case of the Clairaut equation. However, we have the following example:

Example 2.4. $y=x \cdot p^{2}+p^{3}$. We can calculate that $\Sigma_{\pi}\left(E^{2}\right)=\{(x, y, p) \mid y=$ 
$x \cdot p^{2}+p^{3}$ and $\left.p \cdot(2 x+3 p)=0\right\}$ and $\Sigma_{c}\left(E^{2}\right)=\{(x, y, p) \mid y=p=0\}$. We can show that $\Sigma_{c}\left(E^{2}\right)$ is the singular solution in the strict sense.

It follows from the above example that the contact singular set $\Sigma_{c}\left(E^{r}\right)$ is a candidate for the singular solution instead of the $\pi$-singular set $\Sigma_{\pi}\left(E^{r}\right)$.

In $[10,12]$ we have shown the following theorem.

THEOREM 2.5. For an equation $\left(E^{r}, z_{0}\right)$ and a geometric solution $i:\left(L, z_{0}\right) \subset$ $J^{1}\left(\mathbf{R}^{n}, \mathbf{R}\right)$ of $\left(E^{r}, z_{0}\right)$, the following conditions are equivalent.

(1) $i$ is a singular solution of $\left(E^{r}, z_{0}\right)$ in the strict sense and $\left(E^{r}, z_{0}\right)$ is involutory.

(2) There exists a complete solution of $\left(E^{r}, z_{0}\right)$ such that each leaves are transverse to $i$.

(3) Imagei $\subseteq \Sigma_{c}\left(E^{r}\right)$.

This theorem gives a characterization of singular solutions in the strict sense. We can also characterize the complete integrability as a by-product of the above theorem $[10,11]$.

THEOREM 2.6. For an equation $\left(E^{r}, z_{0}\right) \subset J^{1}\left(\mathbf{R}^{n}, \mathbf{R}\right)$, the following are equivalent.

(1) $\left(E^{r}, z_{0}\right)$ is completely integrable.

(2) $\left(E^{r}, z_{0}\right)$ is involutory and $\Sigma_{c}\left(E^{r}\right)=\emptyset$ or $\Sigma_{c}\left(E^{r}\right)$ is an $n$-dimensional submanifold.

We remark that $\Sigma_{c}\left(E^{r}\right)$ is a geometric solution of $\left(E^{r}, z_{0}\right)$ when it is an $n$-dimensional submanifold. So we have the following corollary of Theorems 2.5 and 2.6.

CoRollary 2.7: An equation $\left(E^{r}, z_{0}\right)$ has a singular solution in the strict sense if and only if $\Sigma_{c}\left(E^{r}\right)$ is an n-dimensional submanifold. Moreover, $\Sigma_{c}\left(E^{r}\right)$ is a singular solution in the strict sense.

\section{Singular solutions in the wider sense}

In this section we describe some properties of singular solutions in the wider sense. We remark that if the equation $\left(E^{r}, z_{0}\right)$ is completely integrable then it is involutory (cf. [16]). It follows that we can apply generalized characteristic method to our situations, so that the following proposition is implicitly proved in the classical theory of first order partial differential equations (cf. [16]).

Proposition 3.1. Let $\left(E^{r}, z_{0}\right)$ be a completely integrable equation. If $z_{0}$ is not contact singular, then the equation $\left(E^{r}, z_{0}\right)$ has no singular solution in any sense.

In $[13,15]$ the uniqueness for singular solutions in the wider sense of completely integrable holonomic systems has been proved.

THEOREM 3.2. If a completely integrable holonomic system $\left(E^{n+1}, z_{0}\right)$ has a singular solution $\left(L, z_{0}\right)$ in the wider sense, then $\left(L, z_{0}\right)=\left(\Sigma_{c}\left(E^{n+1}\right), z_{0}\right)$. 
We have the following corollary of Theorems 2.5, 2.6 and 3.2.

CoRollary 3.3. Let $\left(E^{n+1}, z_{0}\right)$ be a completely integrable holonomic system and $i:\left(L, z_{0}\right) \subset J^{1}\left(\mathbf{R}^{n}, \mathbf{R}\right)$ be a geometric solution of $\left(E^{n+1}, z_{0}\right)$. Then the following are equivalent:

(1) $i$ is a singular solution in the wider sense.

(2) $i$ is a singular solution in the strict sense.

We remark that we establish the notion of singular solutions of holonomic systems under the assumption of complete integrability. However, the following example shows that we do not have the answer of the problem to give a correct and rigorous definition of the notion of singular solutions for general equations even now.

Example 3.4. Let $E^{2} \subset J^{1}(\mathbf{R}, \mathbf{R})$ be defined by

$$
F(x, y, p)=y-\frac{1}{2} x p=0, \quad \text { at } z_{0}=0 .
$$

Then $\Sigma_{c}\left(E^{2}\right)=\left\{z_{0}\right\}$, thus $E^{2}$ is not completely integrable at $z_{0}$.

A family of solution of $E^{2}$ is given by $y=c x^{2}$, where $c$ is an arbitrary constant. Since $E^{2}$ is not completely integrable, each one in this family is a singular solution in the wider sense. However, the above family is "a general solution" in the classical sense, so that each one in this family is not "a singular solution" in the classical sense.

In the last part of this survey we may argue the normal form for first order partial differential equations with singular solution in the strict sense up to contact diffeomorphisms. In [12], we have shown the following normal form theorem.

THEOREM 3.5. Let $\left(E^{r}, z_{0}\right)$ be a first order partial differential equation with singular solution in the strict sense. Then there is a contact diffeomorphism germ

$$
f:\left(J^{1}\left(\mathbf{R}^{n}, \mathbf{R}\right), z_{0}\right) \rightarrow\left(J^{1}\left(\mathbf{R}^{n}, \mathbf{R}\right), 0\right)
$$

such that $\left(f\left(E^{r}\right), 0\right)=\left\{p_{1}=\cdots=p_{d-1}=y=0\right\}$, where $d=2 n+1-r$.

\section{REFERENCE}

[1] C. Carathéodory, Calculus of Variations and Partial Differential Equations of First Order, Part I, Partial Differential Equations of the First Order, Holden-Day, 1965.

[2] A.C. Clairaut, Solution de plusieurs problems, Histore de l'Academie royale de Sciences, Paris (1734), 196-215.

[ 3 ] R. Courant and D. Hilbert, Methods of mathematical physics I, II, Wiley, New York, 1962.

[ 4 ] A.R. Forsyth, A Treatise on differential equations, Macmillan and Co, 1885.

[ 5 ] A.R. Forsyth, Theory of differential equations, Part III partial differential equations, Cambridge Univ. Press, London, 1906.

[6] M. Fukuda and T. Fukuda, Singular solutions of ordinary differential equations, The Yokohama Math. Jour. 15 (1977), 41-58.

[ 7 ] E.L. Ince, Ordinary differential equations, Dover, 1926.

[ 8 ] S. Izumiya, Generic properties of first order partial differential equations, Topology Hawaii (ed. K H Dovermann), World Scientific, Singapore (1991), 91-100. 
[9] S. Izumiya, The theory of Legendrian unfoldings and first order differential equations, Proceedings of the Royal Society of Edinburgh 123A (1993), 517-532.

[10] S. Izumiya, Singular solutions of first order differential equations, Bull. London Math. Soc. 26 (1994), 69-74.

[11] S. Izumiya, A characterization of complete integrability for partial differential equations of first order, Annals of Global Analysis and Geometry 12 (1994), 3-8.

[12] S. Izumiya and B. Li, Overdetermined systems of first order differential equations with singular solution, preprint.

[13] S. Izumiya and J. Yu, How to define singular solutions, Kodai Math. Jour. 16 (1993), 227-234.

[14] M. Kossowski, First order partial differential equations with singular solution, Indiana Univ. Math. Jour. 35 (1986), 209-223.

[15] B. Li and S. Izumiya, On singular solutions of systems of first-order partial differential equations, Kobe Journal of Mathematics (to appear).

[16] V.V. Lychagin, Local classification of non-linear first order partial differential equations, Russian Math. Surveys 30 (1975), 105-175.

[17] I.G. Petrovski, Ordinary differential equations, Prentice-Hall, 1966.

\author{
S. IZUMIYA \\ Department of Mathematics \\ FACULTY OF SCIENCE \\ HOKKAIDO UNIVERSITY \\ SAPPORO 060, JAPAN \\ B. LI \\ Department of Mathematics \\ Changsha Normal University of \\ Changsha, 410007, P.R. China \\ J. YU \\ INSTITUTE OF MATHEMATICS \\ ACADEMIa Sinica \\ BeiJing 100080, P.R. China
}

WATER RESOURCES AND Electric POWER 